\title{
PSYCHOLOGICA
}

\section{Primeiros Socorros Psicológicos: intervenção psicológica na catástrofe}

Autor(es): $\quad$ Beja, Maria João; Portugal, Alda; Câmara, Joana; Berenguer, Cláudia;

Rebolo, Ana; Crawford, Carlota; Gonçalves, Dinis

Publicado por: Imprensa da Universidade de Coimbra

URL

persistente: URI:http://hdl.handle.net/10316.2/43574

DOI: $\quad$ DOI:https://doi.org/10.14195/1647-8606_61-1_7

Accessed : $\quad$ 26-Apr-2023 11:42:16

A navegação consulta e descarregamento dos títulos inseridos nas Bibliotecas Digitais UC Digitalis, UC Pombalina e UC Impactum, pressupõem a aceitação plena e sem reservas dos Termos e Condições de Uso destas Bibliotecas Digitais, disponíveis em https://digitalis.uc.pt/pt-pt/termos.

Conforme exposto nos referidos Termos e Condições de Uso, o descarregamento de títulos de acesso restrito requer uma licença válida de autorização devendo o utilizador aceder ao(s) documento(s) a partir de um endereço de IP da instituição detentora da supramencionada licença.

Ao utilizador é apenas permitido o descarregamento para uso pessoal, pelo que o emprego do(s) título(s) descarregado(s) para outro fim, designadamente comercial, carece de autorização do respetivo autor ou editor da obra.

Na medida em que todas as obras da UC Digitalis se encontram protegidas pelo Código do Direito de Autor e Direitos Conexos e demais legislação aplicável, toda a cópia, parcial ou total, deste documento, nos casos em que é legalmente admitida, deverá conter ou fazer-se acompanhar por este aviso. 
VOLUME

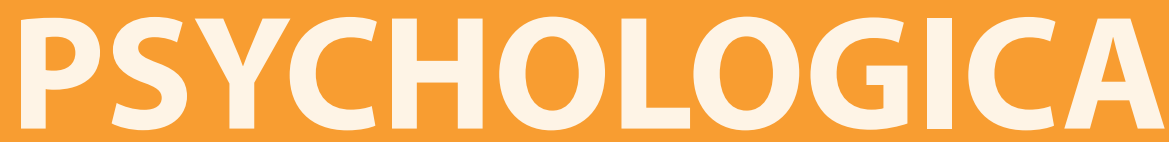

IMPRENSA DA UNIVERSIDADE DE COIMBRA

COIMBRA UNIVERSITY PRESS

FACULDADE DE PSICOLOGIA E DE CIÊNCIAS

DA EDUCAÇÃO DA UNIVERSIDADE DE COIMBRA 


\title{
Primeiros Socorros Psicológicos: Intervenção psicológica na catástrofe
}

\author{
Maria João Beja ${ }^{1}$, Alda Portugal ${ }^{2}$, Joana Câmara ${ }^{3}$, Cláudia Berenguer ${ }^{4}$, \\ Ana Rebolo ${ }^{5}$, Carlota Crawford ${ }^{6}$ e Dinis Gonçalves ${ }^{7}$
}

Psychological First Aid: Psychological intervention in disaster

\begin{abstract}
Catastrophes can be defined as traumatic incidents conducting to major life, health and or material losses, affecting many individuals. The increasing occurrence and magnitude of these incidents led to the consciousness of its impact on psychological functioning and mental health have promoted the development of structured and organized interventions. Psychological First Aid joints several principles and guidelines in an early catastrophe intervention intending the promotion of emotional and psychological stability in individuals affected by critical incidents. This article intends to contribute to the theoretical systematization in an emerging study field in Portugal.
\end{abstract}

Keywords: Disaster; Psychosocial intervention; Psychological first aid; RAPID-PFA model.

1 Universidade da Madeira. Email: mjoao@uma.pt

2 Universidade da Madeira. Email: alda.portugal@staff.uma.pt

3 Mestrado em Neuropsicologia da Universidade Católica Portuguesa. Email: joana.fcamara@gmail.com

4 Mestrado Integrado em Psicologia Clínica do ISPA. Email: claudia.berenguer@hotmail.com

5 Mestrado Integrado em Psicologia Clínica do ISPA. Email: catarinarebolo@hotmail.com

6 Mestrado da Educação da Universidade da Madeira. Email: carlota_mana_7@hotmail.com

7 Licenciatura em Psicologia da Universidade da Madeira. Email: dinis97live@gmail.com 


\section{Resumo}

As catástrofes são acontecimentos traumáticos que provocam grandes perdas de vida, com implicações na saúde e/ou materiais que podem afetar muitas pessoas. O incremento da ocorrência e magnitude destes incidentes conduziu a uma consciencialização crescente sobre o impacto das mesmas ao nível do funcionamento psicológico e da saúde mental, assim como da importância do desenvolvimento de uma intervenção precoce cuidadosamente estruturada e organizada. Os Primeiros Socorros Psicológicos foram desenvolvidos como uma forma de intervenção de apoio psicossocial precoce e englobam um conjunto de princípios e diretrizes orientadas para a promoção da estabilização emocional em indivíduos afetados por incidentes críticos. Este artigo pretende contribuir para a sistematização dos conhecimentos nesta área de estudos que se encontra numa fase emergente em Portugal.

Palavras-chave: catástrofe; intervenção psicossocial; primeiros socorros psicológicos; modelo RAPID-PFA

\section{INTRODUÇÃO}

As catástrofes podem ser naturais ou provocadas pelo Homem, climáticas ou tecnológicas, expetáveis ou imprevisíveis, e afetam necessariamente um vasto leque de sujeitos a nível material, físico e psicológico (Franco, 2015). Os eventos críticos, provavelmente mais frequentes nas atuais circunstâncias ambientais e climáticas, por um lado, e sociopolíticas, por outro, apresentam-se como períodos pautados pela instabilidade e desequilíbrio físico e psíquico. Acontecimentos como catástrofes naturais, conflitos armados, terrorismo, epidemias suscitam uma miríade de reações desadaptativas que podem vir a comprometer a integridade biopsicossocial do ser humano, pelo que urge fazer face a esses fenómenos de cariz multivariável e auxiliar os seres humanos que os vivenciam (Inter-Agency Standing Commitee, 2007).

A intervenção na catástrofe contempla uma vasta panóplia de metodologias e técnicas que operam no sentido de potenciar na vítima a resiliência através do desenvolvimento de mecanismos protetores e de perceções de autoeficácia, que lhe permitam: (a) lidar com o evento traumático, (b) promover a recuperação e a reorganização do seu funcionamento nos diferentes contextos e (c) diminuir a probabilidade de evolução para quadros psicopatológicos severos (Ohio Mental Health \& Addiction Services, 2013). 
Os Primeiros Socorros Psicológicos constituem uma abordagem de intervenção na catástrofe que visa ajudar os indivíduos na minoração do stress emocional, no desenvolvimento e na adoção de estratégias de coping adaptativas que concedam ao indivíduo a possibilidade de recuperar, ainda que parcialmente, o seu funcionamento físico, cognitivo, emocional e social prévio ao acontecimento catastrófico (National Child Traumatic Stress Network, 2006; Ohio Mental Health \& Addiction Services, 2013).

O presente trabalho tem como principais objetivos fazer um breve enquadramento histórico sobre os Primeiros Socorros Psicológicos, identificar os seus aspetos instrumentais e multidisciplinares, bem como os seus elementos nucleares de operacionalização. Pretende-se, ainda, explorar os modelos de intervenção e as suas vantagens, assim como aludir a algumas exigências de índole comportamental, ética e deontológica que se colocam ao nível da prestação dos Primeiros Socorros Psicológicos. Por fim, serão consideradas algumas questões associadas à validação da eficácia dos Primeiros Socorros Psicológicos junto dos indivíduos que experienciaram situações-limite.

\section{Enquadramento Histórico}

Não é possível identificar com exatidão o momento em que se iniciou a intervenção psicológica nas catástrofes, contudo, estima-se que tenha sido em meados do século XX (Franco, 2015). Em 1909, Stierlin publicou um estudo intitulado Psycho-neuropathology as a Result of a Mining Disaster, no qual apresentou a teoria de intervenção em catástrofes aplicada numa ocorrência concreta: a explosão de uma mina de carvão, em França, que havia ocorrido três anos antes (Mitchell \& Everly, 1995 citado por Franco, 2015). Em 1944, a partir do trabalho levado a cabo com sobreviventes de um incêndio em Boston em 1943, fatal para 500 pessoas, Lindemann foi o primeiro a explorar a intervenção psicológica nos momentos que se sucedem a uma catástrofe. Intitulou a sua pesquisa The Sympthomatology of Management of Acute Grief, na qual abordou elementos caracterizadores do luto e possíveis reações típicas de um sobrevivente a uma catástrofe (Franco, 2015). Porém, é apenas no final da $2^{\text {a }}$ Guerra Mundial que emerge pela primeira vez a designação de Primeiros Socorros Psicológicos, não como a conhecemos hoje, mas como técnica de debriefing psicológico, estratégia que se foca na expressão de sentimentos e no relato da situação traumática (Fox et al., 2012). Mais tarde, Thorne (1952, citado por Everly, Perrin \& Everly, 2008) assinalou que a capacidade para reconhecer rápida e prontamente os indícios reveladores de sequelas psicológicas disruptivas era um alicerce para uma intervenção psicológica eficaz. Este autor 
propôs que as intervenções, no contexto de incidentes críticos, devessem abarcar técnicas como a estabilização, a catarse e o aconselhamento. Posteriormente, em 1954, os Primeiros Socorros Psicológicos foram referenciados numa monografia publicada pela Associação Americana de Psiquiatria (Everly, Barnett \& Links, 2012). A partir de então seguiu-se um período em que foram praticamente inexistentes as pesquisas no âmbito dos Primeiros Socorros Psicológicos. Nas décadas de 70 e 80 foi desenvolvido, em contexto militar, um modelo amplamente adotado por profissionais de saúde, bombeiros e polícias para fazer face a situações de catástrofe e emergência, o Critical Incident Stress Management (CISM), que albergava uma componente designada Critical Incident Stress Debriefing (CISD) (Uhernik \& Husson, 2009; Van Emmerik, Kamphuis, Hulsboch \& Emmelkamp, 2002). A partir da década de 80 , assistiu-se a uma forte tendência para explorar novas formas e estratégias de intervenção psicológica no quadro das catástrofes. Foi em meados da década de 90 que começaram a surgir investigações que aludiam à diminuta eficácia do CISD e às repercussões negativas associadas à sua implementação. Considerava-se que esta ferramenta de intervenção não só não reduzia a sintomatologia característica da Perturbação de Stress Pós-Traumático e da Perturbação Depressiva Major, como poderia potenciar a "retraumatização" dos sobreviventes e prestadores de apoio dado a sua exposição à "ventilação catártica" de sentimentos e emoções (Raphael, Meldrum \& McFarlane, 1995; Rose, Bisson, Churchill \& Wessely, 2005; Van Emmerik et al., 2002).

Em 1991 foi desenvolvido pela Cruz Vermelha o Centro de Apoio Psicológico de Copenhaga, constituindo-se como um centro de referência ao nível do trabalho desenvolvido na área das catástrofes. Um dos seus principais objetivos consistia no fornecimento de orientações à Federação Internacional e às Sociedades Nacionais da Cruz Vermelha (Cherpitel, 2001 citado por Franco, 2015). Em 1991 a Inter-Agency Standing Committee (IASC) foi fundada com o propósito basilar de dar resposta a situações de catástrofe e emergência (Fox et al., 2012; Everly et al., 2012). Em 1992, a IASC foi aprovada pela Assembleia Geral das Nações Unidas com a finalidade de estabelecer a coordenação entre as agências de ajuda humanitária, promovendo a eficácia da ajuda humanitária, nomeadamente através da partilha de recursos e conhecimentos na área do apoio psicossocial em situações de emergência (Fox et al., 2012; Everly et al., 2012; IASC, 2007). Mais tarde, a comunidade internacional dedicou-se à construção e implementação de diretrizes respeitantes aos Primeiros Socorros Psicológicos, gradualmente consagrados como dimensão central de intervenção junto de vítimas de incidentes críticos. Com os atentados de 11 de Setembro de 2001 em Nova Iorque, verificou-se um novo e mais intenso impulso a nível das investigações, desenvolvimento de diretrizes e operacionalização dos Primeiros Socorros Psicológicos no quadro da intervenção psicológica na crise (Shultz \& Forbes, 2014). 
Atualmente, os Primeiros Socorros Psicológicos têm vindo a ser alvo de um amplo escrutínio, sendo amplamente recomendados por múltiplas organizações e entidades internacionais de renome, como a Organização Mundial de Saúde (OMS), a Federação Internacional da Cruz Vermelha, o National Center for Post Traumatic Stress Disorder (NCPTS), o Disaster Mental Health Institute of the University of South Dakota, as Red Crescent Societies, entre outras (Fox et al., 2012; McCabe et al., 2014). Além disto, as crescentes ameaças e ataques terroristas (e.g., Nova Iorque, Londres, Madrid), veiculados ou difundidas pelos serviços de comunicação social, vieram reforçar a necessidade de aprofundar esta temática (Fischer \& Ali, 2008).

\section{Aspetos instrumentais e multidisciplinaridade dos Primeiros Socorros Psicológicos}

Os Primeiros Socorros Psicológicos representam uma abordagem de apoio psicossocial dirigida a grupos de indivíduos e/ou comunidades afetadas por situações de catástrofe, de origem natural ou humana (National Child Traumatic Stress Network, 2006). Pretende-se que a atuação, ao nível dos Primeiros Socorros Psicológicos junto das vítimas, seja o mais precoce possível, no sentido de reduzir o stress causado pelos eventos traumáticos e, assim, promover um funcionamento adaptativo a curto e a longo prazo (National Child Traumatic Stress Network, 2006). Os Primeiros Socorros Psicológicos incluem a recolha de informação básica, que permita a realização de avaliações rápidas sobre as necessidades e preocupações imediatas dos sobreviventes; compreendem o conjunto de estratégias de sinalização precoce de indícios reveladores de disfunção despoletados pelo episódio crítico, tendo por objetivo a inviabilização ou, pelo menos, a mitigação da progressão de tais indícios para condições crónicas; consistem, ainda, numa resposta de cariz humanitário, marcada pela compaixão e solidariedade, cujo enfoque reside na prestação de auxílio e suporte psicossocial não intrusivo e consentido (Australian Psychological Society, 2013; Bradel \& Bell, 2014; National Child Traumatic Stress Network, 2006; The Sphere Project, 2011; World Health Organization, 2013). De facto, os Primeiros Socorros Psicológicos mobilizam aspetos básicos e fundamentais da experiência humana, podendo ser concetualizados como uma forma de operacionalização do "bom senso comum" (Shultz \& Forbes, 2014).

Os Primeiros Socorros Psicológicos não constituem um método de diagnóstico, uma intervenção psicoterapêutica, uma forma de tratamento, nem tão pouco um modo de debriefing psicológico ou alguma espécie de interrogatório de caráter mais invasivo; em suma, não almejam substituir a intervenção terapêutica. Por isso mesmo, não se colocam exigências rígidas quanto à seleção dos agentes que procedem à aplicação dos Primeiros Socorros Psicológicos, pelo que esta pode ser 
realizada tanto por equipas de profissionais que intervêm em situações de crise, como por indivíduos com formações de base variadas, desde que devidamente treinados (Ohio Mental Health \& Addiction Services, 2013; World Health Organization, World Vision International \& The United Nations Children's Fund, 2014).

Um aspeto fulcral a ter em consideração é o local e as características das infraestruturas onde os Primeiros Socorros Psicológicos são providenciados. Idealmente, estas devem ser seguras, minimamente confortáveis, espaçosas e resguardadas, i.e., distantes de quaisquer estímulos visuais ou sonoros provenientes do episódio crítico (Australian Psychological Society, 2013). Se nem sempre for possível reunir as condições enunciadas, devem ser ressalvados pelo menos os atributos da segurança e da distância mínima em relação ao local afetado. Alguns dos locais mais recorrentes para a prestação dos Primeiros Socorros Psicológicos são os centros comunitários, centros de saúde, escolas e outras instituições (World Health Organization, War Trauma Foundation \& World Vision International, 2011). Um dos pressupostos subjacentes aos Primeiros Socorros Psicológicos é o de que todos os seres humanos são detentores de aptidões intrínsecas de coping que lhes permitem fazer face às adversidades, bem como superar os desafios impostos pelos eventos iminentemente traumáticos. Assim, a existência de um contexto favorável que reúna as condições requeridas para a satisfação das necessidades elementares do indivíduo, consonante com as suas especificidades, acaba por revelar-se como condição indispensável para o fomento e o desenvolvimento de tais capacidades (Snider, Chehil, \& Walker, 2012; World Health Organization, 2013).

No contexto específico da intervenção psicológica em circunstâncias de catástrofe, Erra e Mouro (2014) reforçam a importância de um compromisso entre as diferentes entidades públicas e privadas, assim como a necessidade de elaboração de um plano nacional de atuação especificamente concebido para fazer face a situações desta natureza. As autoras consideram fulcral que haja uma intervenção sistémica e articulada entre os diferentes organismos, nomeadamente a proteção civil, os bombeiros, as forças policiais e de segurança, as equipas médicas e psicossociais e a segurança social (Autoridade Nacional de Proteção Civil, 2013; Erra \& Mouro, 2014). A intervenção multidisciplinar, pautada pela interdependência e complementaridade, conduz à edificação de uma resposta consolidada e eficaz no domínio das situações-limite. Suplementarmente, a definição e planeamento de uma resposta de emergência que compreenda estas particularidades requer um conhecimento prévio dos recursos locais preexistentes, aliado à aferição da sua acessibilidade por parte dos elementos integrantes da comunidade envolvente (Erra \& Mouro, 2014).

Deste modo, na sequência de uma catástrofe é necessário adotar como ponto de referência um conjunto de procedimentos operacionais padronizados que conste de um plano nacional de emergência que, por sua vez, contemple seis estádios de 
intervenção indispensáveis, designadamente: a mobilização de equipas médicas e de apoio psicossocial para os locais afetados; a prestação de suporte socio emocional individualizado a par da administração de Primeiros Socorros Psicológicos (às vítimas primárias, secundárias e terciárias); a evacuação da população de risco e de especial vulnerabilidade, mais concretamente crianças, adolescentes, grávidas, idosos, pessoas com necessidades especiais e doentes; a disponibilização de apoio logístico à população-alvo por via da distribuição de bens de primeira necessidade (mantimentos, agasalhos, abrigo) e do suprimento das suas necessidades elementares (fisiológicas e de segurança) e, se necessário, da prestação de cuidados médicos imediatos; a realização de operações de busca e/ou de reencaminhamento de pessoas desaparecidas para os seus familiares; e, por fim, o reconhecimento de vítimas mortais e subsequente prestação de suporte psicológico particularizado aos familiares das vítimas (Autoridade Nacional de Proteção Civil, 2013; Erra \& Mouro, 2014; IASC, 2007; World Health Organization, War Trauma Foundation \& World Vision International, 2011).

Em suma, podemos constatar que os Primeiros Socorros Psicológicos se traduzem num processo complexo, multifacetado e transdisciplinar de resposta em situações de catástrofe em que se torna fundamental a articulação da intervenção estritamente psicológica com as demais vertentes disciplinares e operacionais, quer no cenário pós-catástrofe, quer no domínio da prevenção (Autoridade Nacional de Proteção Civil, 2013).

\section{Operacionalização da Intervenção}

\section{Elementos nucleares dos Primeiros Socorros Psicológicos}

Segundo Uhernik e Huson (2009) e Allen et al. (2010), os princípios e técnicas que subjazem aos diversos modelos de Primeiros Socorros Psicológicos atendem a quatro pré-requisitos centrais: (a) serem consistentes com as evidências empíricas em matéria de risco e resiliência no seguimento de experiências traumáticas; (b) serem passíveis de implementação em contextos distintos; (c) adequarem-se a diversas faixas etárias; (d) e, finalmente, incorporarem na sua intervenção as especificidades culturais por forma a serem administrados de modo flexível.

Foram identificados por Hobfoll et al. (2007) cinco elementos nucleares dos Primeiros Socorros Psicológicos: segurança, estabilização emocional, união, autoeficácia e esperança. No que concerne à segurança, é fulcral minimizar ou evitar a exposição a ameaças de natureza diversa daquela que deu azo à situação crítica, 
intervindo aqui as componentes da assistência logística, médica e psicológica, referidas anteriormente. Relativamente à estabilização emocional, esta poderá ser atingida através da disponibilidade para ouvir a história singular de cada indivíduo afetado e para compreender as emoções que o episódio crítico desencadeou, sendo importante que a partilha seja voluntária e não forçada. Quanto à união, é necessário ajudar as vítimas a entrarem em contacto com o seu núcleo familiar e amigos próximos, fornecer informações precisas e pertinentes, além de direcionar as vítimas para os serviços de apoio disponíveis. O técnico de Primeiros Socorros Psicológicos deve, também, promover as competências de autoeficácia do sobrevivente, envolvendo-o ativamente na tomada de decisão e na definição de prioridades ao nível da resolução imediata dos problemas. Por fim, os prestadores de Primeiros Socorros Psicológicos devem transmitir uma perspetiva positiva, otimista e realista sobre a recuperação pós-evento traumático (Hobfoll et al., 2007).

A administração dos Primeiros Socorros Psicológicos deve reger-se por oito princípios centrais aos quais subjazem três componentes basilares da atuação, nomeadamente, a observação, a escuta e a aproximação (Uhernik \& Husson, 2009; World Health Organization, War Trauma Foundation \& World Vision International, 2011), Assim, o prestador de Primeiros Socorros Psicológicos deve, em primeira instância, identificar os indivíduos que experienciaram o evento traumático e iniciar o contacto de forma compassiva e não invasiva, considerando que alguns indivíduos poderão não procurar ou consentir este tipo de ajuda. Neste primeiro contacto, o provedor de Primeiros Socorros Psicológicos deve fazer uma nota introdutória sucinta acerca do seu papel e funções de que está incumbido. Em seguida, afigura-se crucial prover segurança e conforto físico e estabilizar as emoções do sobrevivente, por via da sinalização das necessidades que requerem satisfação prioritária e da normalização do seu sofrimento (explicitação da inevitabilidade das reações e comportamentos despoletados pelo episódio crítico), algo que contribui para o decréscimo dos níveis de stress e preocupação e pode auxiliar em termos da adoção de algumas estratégias de coping. A recolha de informação permite que seja possível priorizar, ou seja, triar e hierarquizar a população que se encontra mais vulnerável, por forma a ser possível intervir com prontidão. A assistência prática consiste na delineação de um plano de ação que faculte ferramentas ao indivíduo para lidar com o acontecimento potencialmente traumático. Através da conexão com a rede social de suporte, é possível auxiliar o indivíduo a estabelecer contacto com os seus familiares e grupo de pares. Em virtude das situações de crise terem um caráter confuso e desorientador passível de obstaculizar as competências que permitem que o indivíduo enfrente as dificuldades, os técnicos que aplicam os Primeiros Socorros Psicológicos devem facultar informação verbal e escrita acerca das estratégias de coping, por forma a munir o indivíduo de ferramentas que lhe 
permitam lidar com o problema, fomentar um processo resiliente e predispor para um funcionamento adaptativo e integrativo. Se eventualmente as informações partilhadas pelo técnico de Primeiros Socorros Psicológicos forem insuficientes, urge reencaminhar o indivíduo para serviços que lhe facultem um suporte mais especializado (Bradel \& Bell, 2014; Uhernik \& Husson, 2009).

\section{Modelos de Intervenção: o modelo RAPID-PFA}

Uma miríade de modelos de Primeiros Socorros Psicológicos, desenhados e estruturados com base numa variedade de critérios e pontos de referência, encontra-se disponível, sendo que, em boa medida, a diferenciação de tais modelos resulta da diferente valorização dos elementos nucleares apresentados anteriormente (Hobfoll et al., 2007; Shultz \& Forbes, 2014). Para além disso, cada modelo é detentor de um conjunto de linhas orientadoras e de ações principais que norteiam a intervenção propriamente dita (Shultz \& Forbes, 2014).

Everly et al. (2012) contribuíram para o desenvolvimento de um dos modelos mais relevantes de Primeiros Socorros Psicológicos intitulado RAPID-PFA (isto é, Reflective Listening, Assessment, Prioritization, Intervention and Disposition - Psychological First Aid). Este modelo contempla cinco estádios primordiais de atuação: o estabelecimento da relação, a avaliação, a priorização, a intervenção e, por fim, a monitorização do progresso ou acompanhamento. Segundo Everly e Flynn (2005), o RAPID-PFA apresenta oito objetivos fundamentais: (1) ampliar a capacidade de compreensão de uma situação problemática e ouvir ativamente; (2) avaliar e priorizar as necessidades básicas das pessoas; (3) reconhecer reações comportamentais e psicológicas benignas em circunstâncias de crise; (4) reconhecer reações comportamentais e psicológicas severas, potencialmente desestabilizadoras e incapacitantes; (5) mitigar o stress agudo com recurso a determinados tipos de intervenções; (6) reconhecer quando é necessário facultar e/ou facilitar o acesso a apoio suplementar ao nível da saúde mental; (7) reduzir potenciais riscos adversos advindos da intervenção e (8) promover a auto-preservação.

O estabelecimento de uma relação empática e harmoniosa entre o técnico de Primeiros Socorros Psicológicos e o sobrevivente, por via da escuta reflexiva e ativa da narrativa do indivíduo afetado, é fundamental na medida em que permitirá a identificação dos aspetos mais relevantes da experiência traumática do indivíduo (McCabe et al., 2014). Assim, o prestador de Primeiros Socorros Psicológicos deve, num primeiro momento, procurar colocar questões abertas e exploratórias que conduzam à compreensão do sucedido e do estado atual do indivíduo (reações associadas) e parafrasear o que é verbalizado pela vítima, de modo a validar e normalizar as suas reações, sentimentos e emoções mais imediatas após o episó- 
dio (Everly, McCabe, Semon, Thompson \& Links, 2014). A avaliação, por sua vez, consiste na identificação das necessidades idiossincráticas do indivíduo, na análise das reações pós-traumáticas expressas, na averiguação da sua natureza funcional ou disfuncional e no apaziguamento do sofrimento e dor agudas. É nesta fase que o prestador de Primeiros Socorros Psicológicos reúne informações pertinentes a respeito dos fatores que podem facilitar ou obstaculizar a recuperação e restabelecimento de um funcionamento adaptativo, como por exemplo as capacidades do indivíduo para compreender e seguir orientações, expressar emoções de um modo saudável e construtivo, adaptar-se ao contexto e aceder a recursos interpessoais (Everly et al., 2014). Importa referir que, para que tal seja possível, o prestador de Primeiros Socorros Psicológicos deverá colocar questões diretivas e específicas por forma a clarificar potenciais ambiguidades e, deste modo, moldar a sua atuação em virtude das particularidades do indivíduo (McCabe et al., 2014). A priorização afigura-se como uma extensão da avaliação, reportando-se à triagem e hierarquização dos indivíduos mais vulneráveis e que devem ser intervencionados com maior prontidão. De um modo geral, trata-se de uma forma de identificar e distinguir as reações benignas e naturais de eventuais reações disfuncionais e mal-adaptativas. Esta priorização é realizada com base numa multiplicidade de indícios, expressos pela vítima, ao nível da sua capacidade cognitiva (dificuldade em recordar os eventos e resolver problemas), comportamental (incapacidade de discernir as consequências dos seus atos e agir impulsivamente, isto é, autoflagelando-se ou causando dano a outrem), expressão emocional e afetiva, adaptabilidade social, recursos interpessoais (competência para funcionar no quotidiano e realizar tarefas elementares, como tratar da higiene e alimentar-se, cuidar de si e daqueles que dependem de si, trabalhar) e a sua disponibilidade e consentimento para ser intervencionada (Everly et al., 2012; McCabe et al., 2014). No que concerne à intervenção, o prestador de Primeiros Socorros Psicológicos deve mitigar o stress agudo, tentar restabelecer as capacidades funcionais do indivíduo por via da adoção de estratégias de coping adaptativas (e.g., técnicas de relaxamento, de respiração, de controlo da raiva e de outras emoções negativas) e da promoção da resiliência, do suporte da sua rede de apoio social e do esclarecimento e antecipação das emoções e sentimentos que o indivíduo irá experienciar nos dias subsequentes ao evento limite. Afirma-se igualmente crucial infundir esperança e uma perspetiva temporal de futuro nos indivíduos afetados, uma vez que em circunstâncias de catástrofe existe uma tendência para agir impulsivamente e tomar decisões precipitadas. De acordo com Everly e Lating (2002, citados por Everly et al., 2012), as metodologias de intervenção cognitivo-comportamentais parecem ter um impacto positivo na diminuição do stress agudo e no restabelecimento da calma. Os autores adiantam que modelos educacionais e exploratórios, como por exemplo o modelo de Cannon designado 'Fight - Flight', 
contribuem para diminuir o impacto nefasto das catástrofes. McCabe et al. (2014) acrescentam que o prestador de Primeiros Socorros Psicológicos pode recorrer a técnicas simples para reduzir o stress agudo, tais como aconselhar, distrair e orientar o indivíduo, e que em função do nível de disfuncionalidade podem ser adotadas abordagens de autorregulação psicofisiológica (isto é, respiração diafragmática) e de reenquadramento cognitivo. Tal permite ao indivíduo estar mais apto a reaver o controlo sobre a sua vida e retomar os seus hábitos quotidianos (Everly et al., 2008; Everly et al., 2014). Finalmente, para estes autores, a monitorização ou acompanhamento afigura-se central para elucidar o técnico de Primeiros Socorros Psicológicos a respeito do quão bem sucedida foi a sua atuação. Uma das formas de avaliar algum do progresso do indivíduo intervencionado consiste no estabelecimento de um contacto posterior e análise do seu funcionamento básico, direcionado para a avaliação da sua capacidade e disponibilidade para levar a cabo as tarefas diárias mais elementares, prosseguir a sua atividade laboral e estabelecer contacto assíduo com os seus amigos e familiares. Se o indivíduo retomar a sua vida de forma bem sucedida, a intervenção do técnico termina (Everly et al. 2012; McCabe et al., 2014). Porém, se o funcionamento do indivíduo continuar a manifestar-se inadaptativo, poder-se-á seguir um segundo follow-up, sendo que a partir do terceiro deverá ser disponibilizado outro tipo de cuidados mais particularizados e que redefinam a abordagem à vítima (Everly et al., 2014).

\section{Benefícios da prestação de Primeiros Socorros Psicológicos}

Inúmeras investigações científicas têm vindo a sublinhar o relevo e os benefícios dos Primeiros Socorros Psicológicos nas etapas iniciais da resposta psicossocial a episódios de catástrofe (McCabe et al., 2014). De facto, segundo Bradel e Bell (2014), os indivíduos expostos a catástrofes naturais (e.g., terramotos, furacões, inundações, deslizamentos de terra) e humanas (e.g., atentados terroristas) estão mais suscetíveis de desenvolver determinados quadros psicopatológicos, sendo os mais recorrentes a Perturbação Depressiva Major e as Perturbações de Ansiedade. Além disso, existem outros fatores que precipitam a manifestação destes e outros quadros psicopatológicos, nomeadamente o grau de exposição e magnitude (severidade) do evento traumático, a necessidade de evacuação e deslocação para um local desconhecido, a perda de um ente querido ou de indivíduos conhecidos (luto que se pode tornar complicado), a ausência de uma rede social de apoio, a presença de problemas de saúde pré-existentes, algumas variáveis sociodemográficas específicas (e.g., género e nível socioeconómico), determinados traços de personalidade (e.g., neuroticismo) e mecanismos de atribuição de significado ao acontecimento traumático menos adaptativos (Bradel \& Bell, 2014; Franco, 2012; Slaikeu, 1990). 
Paralelamente, Bradel e Bell (2014) realçam que os incidentes críticos de caráter ansiógeno e stressante desencadeiam frequentemente alterações bioquímicas singulares, por exemplo, o aceleramento do ritmo cardíaco e a libertação de adrenalina após a estimulação do sistema nervoso simpático, que predispõem o ser humano para a reação profícua (fight), ou para a fuga (flight). As circunstâncias propiciadoras de stress agudo podem induzir reações de índole fisiológica (e.g., espasmos e dores musculares, comprometimento do sistema imunitário), comportamental (e.g., perturbações alimentares e do sono, fobias) cognitiva (e.g., declínio da atenção seletiva, estados de confusão, incapacidade de resolução de problemas), emocional (e.g., tristeza, raiva, medo), e espiritual (e.g., emergência de questões existenciais multitemáticas: o significado da vida, a intervenção de entidades superiores, o destino) que, por vezes, obstaculizam o desempenho do indivíduo na sua vida quotidiana (Bradel \& Bell, 2014; National Child Traumatic Stress Network, 2006).

Neste sentido, e para colmatar toda esta diversidade de impactos potenciais do episódio crítico, os Primeiros Socorros Psicológicos têm como finalidade munir o indivíduo de competências úteis que lhe permitam aprender e desenvolver padrões de resposta adaptativos perante circunstâncias stressantes, por forma a fomentar a resiliência e a perseverança (Autoridade Nacional de Proteção Civil, 2013; Chan, Chan, \& Kee, 2012). Neste sentido, o constructo de autoeficácia, proposto por Bandura, desempenha um papel central no que diz respeito à eficácia das intervenções. Trata-se de um constructo microanalítico referente às crenças e perceções que um indivíduo tem a respeito das suas competências para desempenhar eficientemente uma tarefa e, por conseguinte, atingir o resultado ambicionado independentemente dos obstáculos que possam surgir. As crenças de autoeficácia revelam-se cruciais para a manutenção da continuidade do esforço e da perseverança em circunstâncias funestas e, como tal, são determinantes na gestão pessoal das consequências da situação traumática (Graham \& Weiner, 1996). Com efeito, segundo a Australian Psychological Society (2013), as crenças de autoeficácia predizem desfechos mais favoráveis, sendo que os indivíduos mais otimistas, detentores de pensamentos mais positivos, que creem ser capazes de ultrapassar as contrariedades que se interpõem na sua jornada, estão aptos a recuperar mais facilmente das repercussões nefastas advindas da situação traumática. A promoção da autoeficácia de cada indivíduo afigura-se, então, como uma tarefa central para a potenciação da intervenção (Hobfoll et al., 2007).

\section{Considerações deontológicas e perfil do prestador de Primeiros Socorros Psicológicos}

A prestação de auxílio em momentos críticos deve ser baseada num conjunto de diretrizes e princípios orientadores de natureza ética e deontológica. No qua- 
dro da necessária salvaguarda da dignidade e dos direitos individuais das pessoas afetadas, urge que o técnico de Primeiros Socorros Psicológicos se despoje de todo o tipo de preconceitos e se iniba de qualquer espécie de condenação ou censura relativamente a sentimentos, emoções e atitudes experienciados aquando da situação-limite (Australian Psychological Society, 2013; National Child Traumatic Stress Network, 2006). Outro aspeto a considerar é a confidencialidade e o sigilo das informações partilhadas. Sublinhe-se ainda que a prestação dos Primeiros Socorros Psicológicos deve estar alinhada com as matrizes socioculturais específicas da população afetada, devendo adaptar-se ao contexto sociocultural e étnico em que a intervenção decorre, por exemplo, na adequação da indumentária, no domínio da língua predominante nas populações intervencionadas, na sensibilidade em relação a hábitos e costumes locais e a rituais comportamentais (e.g., perceber se existe alguma objeção no que toca a determinadas formas de contacto corporal, como segurar a mão) e no conhecimento das crenças culturais e religiosas de cada grupo étnico. Estas considerações poderão facilitar ao técnico uma intervenção válida e não invasiva (World Health Organization, War Trauma Foundation \& World Vision International, 2011; Ohio Mental Health \& Addiction Services, 2013; The Sphere Project, 2011).

Os prestadores de Primeiros Socorros Psicológicos devem deter alguns atributos individuais singulares e obedecer a certos requisitos em termos de perfil pessoal. Em primeiro lugar, devem estar preparados para se submeterem a circunstâncias físicas, emocionais e psicologicamente desafiantes e para intervir em cenários caóticos, pautados pela desolação, destruição, ferimentos alheios e/ou perdas humanas (McCabe et al., 2014; World Helath Organization, World Vision International \& The United Nations Children's Fund, 2014). Consequentemente, teste tipo de ambiente hostil pode revelar-se potencialmente desestabilizador e pernicioso, afetando de modo direto ou indireto tanto o técnico quanto o seu sistema familiar. Como tal, é fundamental que o prestador de Primeiros Socorros Psicológicos goze de boa saúde física e mental e não tenha sido alvo de tratamentos, cirurgias ou restrições dietéticas que comprometam o seu desempenho, nem tenha estado sujeito a experiências de vida emocionalmente exigentes e impactantes (McCabe et al., 2014; World Health Organization, War Trauma Foundation \& World Vision International, 2011). O técnico de Primeiros Socorros Psicológicos deve reagir profícua e adaptativamente aos acontecimentos stressantes e desgastantes com os quais se depara, contrariando estados como a fadiga, a frustração, a resignação, a desmoralização, a alienação e o alheamento, entre outros. Para que isso seja possível, é fundamental autopreservar-se, algo que se torna particularmente exequível através de atributos como a tolerância, a flexibilidade, a paciência e a sensatez, e de hábitos quotidianos promotores da saúde e bem-estar, designadamente ter uma alimentação equilibrada, hábitos de sono 
saudáveis, praticar exercício físico, investir nas relações familiares e interpessoais de modo a desenvolver vínculos sólidos e inquebrantáveis, procurar ter experiências pessoais enriquecedoras e gratificantes, reservar algum tempo para a reflexão, entre outros (Ohio Mental Health \& Addiction Services, 2013; World Health Organization, 2013). Saliente-se, por fim, que a organização que enquadra a intervenção do técnico de Primeiros Socorros Psicológicos tem um papel ativo e determinante na sua satisfação, equilíbrio e bem-estar, pelo que esta deve providenciar formação profissional ao nível da gestão do stress, facultar informações de vária ordem a respeito dos benefícios da função que está a ser desempenhada, encorajar as pausas e promover a rotatividade (World Health Organization, War Trauma Foundation \& World Vision International, 2011; National Child Traumatic Stress Network, 2006).

\section{Evidência Científica da Eficácia}

Ramirez et al. (2013) levaram a cabo uma investigação que consistiu na conceção de uma modalidade de intervenção alternativa radicada nos Primeiros Socorros Psicológicos, denominada Listen Protect Connect (LPC). Esta abordagem foi implementada em contexto escolar, sob administração dos professores, com o objetivo de sinalizar crianças (vítimas de ou alvo de um evento catastrófico) que manifestassem sintomas depressivos e indicativos de Perturbação de Stress Pós-traumático e de Perturbação Depressiva Major, no sentido de atuar ulteriormente para fomentar o desenvolvimento de estratégias de coping adaptativas e mobilizar recursos de saúde mais especializados suscetíveis de inibir a progressão do trauma. Os resultados obtidos demonstraram que a LPC foi uma intervenção eficaz na redução do sofrimento psicológico e incrementou a união e suporte interpessoal no contexto escolar. Porém, é de assinalar que a LPC requer sustentação empírica que estabeleça a sua eficácia e validade, tanto a curto quanto a longo prazo, nos diversos contextos de atuação.

Dieltjens, Moonens, Van Praet, De Buck e Vandekerckhove (2014) efetuaram uma revisão sistemática da literatura com o propósito de identificar o quão válidas e profícuas eram as diretrizes, orientações e modalidades de intervenção realizadas com recurso aos Primeiros Socorros Psicológicos no domínio das catástrofes, situações de emergência e outros eventos de caráter traumático. Os resultados reportaram a insuficiência de evidências científicas fidedignas passíveis de comprovar os benefícios e eficácia dos Primeiros Socorros Psicológicos a longo prazo. Saliente-se que a recolha da evidência empírica relativa à eficácia dos Primeiros Socorros Psicológicos se revela problemática em função da própria natureza das situações de crise, que dificilmente configuram um campo de observação controlado e propício a operações de recolha sistemática de elementos empíricos. 
A escassez de evidências científicas passíveis de ilustrar a eficácia dos Primeiros Socorros Psicológicos não inibe inúmeros especialistas e entidades internacionais de relevo, para além de diversas revisões da literatura, de enfatizar as suas vantagens e benefícios e defender a sua aplicação. Ainda assim, parecem inequívocas as fragilidades dos Primeiros Socorros Psicológicos do ponto de vista da sua validade e sustentabilidade empírica, sendo sobretudo nesse campo que se manifestam atualmente maiores lacunas a nível da pesquisa e investigação adicional (Fox et al., 2012). Um dos pouco exemplos deste tipo de pesquisa é o estudo de Allen et al. (2010), em que foram avaliadas as percepções da eficácia dos Primeiros Socorros Psicológicos por parte dos próprios prestadores de Primeiros Socorros Psicológicos, nos casos em que sucederam os furacões Katrina, Gustav e Ike, e baseados no manual National Child Traumatic Stress Network/National Center for Post Traumatic Stress Disorder Psychological First Aid. Os resultados indicaram que as suas perceções eram predominantemente positivas e que, para além do mais, os técnicos de Primeiros Socorros Psicológicos se sentiram mais confiantes na prestação de cuidados a adultos do que a crianças, o que sugere a necessidade de se diferenciarem modelos de intervenção para as crianças.

Shultz e Forbes (2014) abordam o problema da mensuração da eficácia dos Primeiros Socorros Psicológicos e propõem uma análise comparativa diacrónica dos percursos de indivíduos intervencionados e não intervencionados, por forma a estabelecer um paralelo entre si e identificar as principais divergências. No âmbito da sua tentativa de fixação de procedimentos suscetíveis de consagrar a validação empírica da eficácia dos Primeiros Socorros Psicológicos, os autores realçam a necessidade de assegurar a comparabilidade dos dados (os estudos comparativos devem incidir em indivíduos submetidos aos mesmos estímulos e expostos às mesmas catástrofes), bem como de diversificar os instrumentos de avaliação/mensuração.

\section{CONSIDERAÇÕES FINAIS}

Os Primeiros Socorros Psicológicos inscrevem-se num complexo interdisciplinar, sistémico e complementar de resposta orientado para intervir em cenários de crise, catástrofe e emergência. A singularidade destes contextos de intervenção faz com que seja crucial cultivar competências que habilitem os prestadores de Primeiros Socorros Psicológicos a maximizar a eficácia da sua resposta. Neste quadro, os Primeiros Socorros Psicológicos adquirem configurações sempre distintas em função da especificidade da situação em concreto e das necessidades da população-alvo o que, por sua vez, apela a uma capacidade basilar de modulação e adaptação da 
intervenção, de resto igualmente imposta pelo imperativo deontológico que apela ao seu enquadramento no ambiente cultural prevalecente nos locais intervencionados.

Apesar de os Primeiros Socorros Psicológicos serem amplamente defendidos por toda uma diversidade de agentes e instituições especializadas, subsistem limitações ao nível da mensuração e aferição da sua eficácia e repercussões benéficas a longo prazo, pelo que investigações científicas adicionais afirmam-se determinantes para a sustentação empírica e validação desta ferramenta humanitária. No contexto português, salienta-se o importante papel que a Ordem dos Psicólogos Portugueses (OPP) desempenhou ao criar e disponibilizar uma formação sobre Intervenção Psicológica em Situações de Catástrofe, iniciada no ano de 2014. O objetivo da OPP é colocar à disposição das entidades de proteção civil, uma bolsa de psicólogos, a nível nacional, formados na intervenção em situação de catástrofe, disponíveis para dar um contributo cívico e que são chamados a atuar quando necessário. No entanto, para além deste esforço assinalável, continua a ser central que a comunidade científica se dedique a este tópico, no sentido de identificar modelos de intervenção eficazes para atuar na população portuguesa. Urge alertar para o aumento da magnitude e da frequência das catástrofes, bem como consciencializar para a necessidade de um auxílio estruturado e premeditado às comunidades afetadas, a um nível quer psicossocial, quer psicopatológico.

Podemos retratar o provedor de Primeiros Socorros Psicológicos como uma espécie de espelho, que reflete e prolonga o universo cultural e intrapsíquico do ser humano em sofrimento, mas que também de algum modo o transforma e molda. Este exercício de reverberação e reenquadramento de uma realidade estilhaçada cumpre um fim último: a integração e regeneração do próprio eu. Neste sentido, os Primeiros Socorros Psicológicos configuram-se como veículos promotores da catarse, do autoconhecimento e da metamorfose da narrativa do indivíduo, concedendo-lhe a possibilidade de retomar - mesmo que de forma periclitante, hesitante e árdua - a vida quotidiana de outrora.

\section{REFERÊNCIAS BIBLIOGRÁFICAS}

Allen, B., Brymer, M. J., Steinberg, A. M., Vernberg, E. M., Jacobs, A., Speier, A. H., \& Pynoos, R. S. (2010). Perceptions of psychological first aid among providers responding to Hurricanes Gustav and Ike. Journal of Traumatic Stress, 23(4), 509-513. doi: 10.1002/jts.20539

Australian Psychological Society (2013). Psychological first aid: An australian guide to supporting people affected by disaster. Retrieved from http://www.redcross.org.au/files/Psychological_First_Aid_An_Australian_Guide.pdf

Autoridade Nacional de Proteção Civil. (2013). Psicologia de catástrofe na gestão de emergência. Boletim Mensal da Autoridade Nacional de Proteção Civil, 63. Retrieved from http://www. prociv.pt/bk/newsletter/PROCIV63_webFinal.pdf 
Bradel, L. T., \& Bell, K. M. (2014). Treatment for survivors of natural disasters. Annals of Psychotherapy \& Integrative Health, 14(1), 1-17.

Chan, A. O. M., Chan, Y. H., \& Kee, J. P. (2012). Improving resistance and resiliency through crisis intervention training. International Journal of Emergency Mental Health, 14(2), 77-87.

Dieltjens, T., Moonens, I., Van Praet, K., De Buck, E., \& Vandekerckhove, P. (2014). A systematic literature search on psychological first aid: Lack of evidence to develop guidelines. PloS one, 9(12), 1-13. doi:10.1371/journal.pone.0114714

Erra, S., \& Mouro, H. (2014). Intervenção do serviço social em contexto de catástrofe: o caso da Madeira 2010. Intervenção Social, 40, 39-58.

Everly, G. S., Jr., \& Flynn, B. W. (2005). Principles and practice of acute psychological first aid after disasters. In G. S. Everly Jr. \& C. L. Parker (Eds.), Mental health aspects of disasters: Public health preparedness and response, revised (pp. 79-89). Baltimore, MD: Johns Hopkins Center for Public Health Preparedness.

Everly, G. S., Barnett, J. D. J., \& Links, J. M. (2012). The Johns Hopkins model of psychological first aid (RAPID - PFA): curriculum development and content validation. International Journal of Emergency Mental Health, 14(2), 95-105.

Everly, G. S., McCabe, O. L., Semon, N. L., Thompson, C. B., \& Links, J. M. (2014). The development of a model of psychological first aid for non-mental health trained public health personnel: The Johns Hopkins RAPID-PFA. Journal of Public Health Management and Practice, 20, 24-29. doi: 10.1097/phh.0000000000000065

Everly, G. S., Perrin, P., \& Everly, G. (2008). Psychological issues in escape, rescue, and survival in the wake of disaster. Mental Health, 12(1), 21-30.

Fischer, P., \& Ai, A. L. (2008). International Terrorism and Mental Health: Recent Research and Future Directions. Journal of Interpersonal Violence, 23(3), 339-361. doi:10.1177/0886260507312292

Fox, J. H., Burkle, F. M., Bass, J., Pia, F. A., Epstein, J. L., \& Markenson, D. (2012). The effectiveness of psychological first aid as a disaster intervention tool: research analysis of peer-reviewed literature from 1990-2010. Disaster Medicine and Public Health Preparedness, 6(03), 247-252. doi: $10.1001 / \mathrm{dmp} .2012 .39$.

Franco, M. H. P. (2015). A Intervenção psicológica em emergências: fundamentos para a prática. São Paulo: Summus Editorial.

Franco, M. H. P. (2012). Crises e desastres: a resposta psicológica diante do luto. O Mundo da Saúde, São Paulo, 36(1), 54-58.

Graham, S., \& Weiner, B. (1996). Theories and principles of motivation. In D.C. Berliner \& R. C. Calfee (1996). Handbook of educational psychology. London: Prentice Hall International

Hobfoll, S. E., Watson, P. E., Bell, C. C., Bryant, R. A., Brymer, M. J., Friedman, M. J., et al. (2007). Five essential elements of immediate and mid- term mass trauma intervention: Empirical evidence. Psychiatry: Interpersonal and Biological Processes, 70, 283-315.

Inter-Agency Standing Committee (2007). IASC guidelines on mental health and psychosocial support in emergency settings. Geneva: IASC.

McCabe, O. L., Semon, N. L., Lating, J. M., Everly Jr, G. S., Perry, C. J., Moore, S. S., Mosley, M. A. M., Thompson, C. B., \& Links, J. M. (2014). An academic-government-faith partnership to build disaster mental health preparedness and community resilience. Public Health Reports, 129(4), 96-106.

McCabe, O. L., Everly Jr, G. S., Brown, L. M., Wendelboe, A. M., Abd Hamid, N. H., Tallchief, V. L., \& Links, J. M. (2014). Psychological first aid: a consensus-derived, empirically supported, 
competency-based training model. American Journal of Public Health, 104(4), 621-628. doi: 10.2105/ajph.2013.301219

National Child Traumatic Stress Network (2006). Psychological first aid: field operations guide.

Ohio Mental Health \& Addiction Services. (2013). A Psychological first aid guide for Ohio colleges and universities: supporting students, faculty and staff. Retrieved from https://www.ohiohighered. org/sites/ohiohighered.org/files/uploads/CampusSafety/Psychological\%20First\%20Aid\%20(2). pdfRamirez, M., Harland, K., Frederick, M., Shepherd, R., Wong, M., \& Cavanaugh, J. E. (2013). Listen protect connect for traumatized schoolchildren: a pilot study of psychological first aid. BMC psychology, 1(1), 26. doi: 10.1186/2050-7283-1-26

Shultz, J. M., \& Forbes, D. (2014). Psychological first aid: RAPID proliferation and the search for evidence. Disaster Health, 2(1), 3-12. doi:10.4161/dish.26006

Slaikeu, K. A. (1990). Crisis intervention: A handbook for practice and research. Boston: Allyn \& Bacon.

Snider, L., Chehil, S., \& Walker, D. (2012). Psychological First Aid. In Pan American Health Organization \& World Health Organization (2012). Mental Health and Psychosocial Support in Disaster Situations in the Caribbean. Retrieved from http://www.paho.org/disasters/index. php?option=com_content\&view=article\&id=1649:mental-health-and-psychosocial-supportin-disaster-situations-in-the-caribbean\&Itemid=807\&lang=en

The Sphere Project (2011). Humanitarian charter and the minimum standards in humanitarian response. United Kingdom: Pratical Action Publishing. doi: 10.3362/9781908176202

Uhernik, J. A., \& Husson, M. A. (2009). Psychological first aid: an evidence informed approach for acute disaster behavioral health response. Compelling Counseling Interventions: VISTAS, 200(9), 271-280.

Van Emmerik, A., Kamphuis, J., Hulsboch, A., \& Emmelkamp, P. (September 2002). Single session debriefing after psychological trauma: A meta-analysis. Lancet, 360 (9335), 766-771.

World Health Organization, War Trauma Foundation \& World Vision International. (2011). Psychological First aid: Guide for fields workers. Retrieved from http://apps.who.int/iris/ bitstream/10665/44615/1/9789241548205_eng.pdf.

World Health Organization, World Vision International, \& The United Nations Children's Fund. (2014). Psychological first aid during Ebola virus disease outbreaks: Facilitator manual (provisional version). World Health Organization: Geneva.

World Health Organization. (2013). Building Back Better Sustainable Mental Health Care after Emergencies. World Health Organization: Geneva. 